\title{
BARREIRAS PARA O DESENVOLVIMENTO DE PROGRAMAS DE ATIVIDADE FISICA PARA IDOSOS: A VISÃO DOS SECRETÁRIOS MUNICIPAIS DE SAÚDE DE SANTA CATARINA
}

\author{
Paula Fabricio Sandreschi ${ }^{1}$ \\ Daniel Rogério Petreça² \\ Giovana Zarpellon $\mathrm{Mazo}^{3}$ \\ Felipe Fank ${ }^{4}$
}

resumo

Objetivo: descrever a visão dos secretários municipais de saúde (SMS) do estado de Santa Catarina (SC) sobre as barreiras para desenvolvimento de programas de promoção de atividade física (AF)

1 Graduada em bacharelado em Educação Física pela Universidade do Estado de Santa Catarina (UDESC). Mestre em Ciências do Movimento Humano pela Universidade do Estado de Santa Catarina (UDESC). Doutoranda do Programa de Pós Graduação em Educação Física da Universidade Federal de Santa Catarina (UFSC). E-mail: paula.sandreski@gmail.com.

2 Graduado em Educação Física pela Universidade do Contestado - Campus Mafra. Especializado em Personal Trainer pela Universidade Federal do Paraná (UFPR). Mestre em Atividade Física e Saúde pela Universidade Federal de Santa Catarina (UFSC). Doutor em Ciências do Movimento Humano pela Universidade do Estado de Santa Catarina (UDESC). E-mail: profdaniel@unc.br.

3 Professora do Departamento de Ciências da Saúde e do Programa de Pós-Graduação em Ciências do Movimento Humano do Centro de Ciências da Saúde e do Esporte da Universidade do Estado de Santa Catarina (CEFID/UDESC). E-mail: giovana.mazo@udesc.br.

4 Graduado em Educação Física pela Universidade do Estado de Santa Catarina (UDESC). E-mail: felipee.fank@gmail.com. 
para idosos e associá-las às características de seu perfil. Método: estudo realizado com SMS de 204 municípios. Foi aplicado questionário com questões sociodemográficas, de formação, de saúde e quatro barreiras para desenvolvimento de programas de AF para idosos, as quais deveriam atribuir um grau de importância. Resultados: a principal barreira considerada como maior importância pelos SMS de SC foi "falta de incentivo financeiro para implantação de ações/atividades relacionadas à AF para idosos" e foi associada ao estado civil, ao nível de atividade física dos secretários e à presença de programa de AF no município. Conclusão: os SMS consideram que a falta de recursos financeiros é uma barreira importante para implementarem programas de AF para idosos e suas características estão associadas à essa percepção.

palavras-chave

Saúde Pública. Administração de Serviços de Saúde. Gestor de Saúde.

A inatividade física, problema recorrente em todo o mundo, é agravada com o envelhecimento populacional. Estima-se que $47 \%$ dos idosos do mundo não praticam atividade física suficiente, conforme recomendado pela Organização Mundial da Saúde (HALLAL et al., 2012). Nas capitais brasileiras, esse valor é maior, podendo chegar a $65,8 \%$ de indivíduos com 65 anos ou mais inativos fisicamente (MS, 2015). Dessa forma, ações para que esse panorama seja revertido são necessárias, uma vez que a prática de atividade física é extremamente importante na prevenção de doenças, manutenção da independência e melhoria da qualidade de vida dessa população (SUN; NORMAN; WHILE, 2013).

Em vista dessa situação, políticas de promoção da atividade física foram incluídas nas agendas mundiais, com destaque para a Estratégia Global de Alimentação Saudável, Atividade Física e Saúde (OMS, 2004). No Brasil, as ações de promoção de atividade física começaram a serem mais incisivas em 2006, com a publicação da Política Nacional de Promoção da Saúde (PNPS) a qual apresentou dentre seus eixos prioritários a prática de atividade física e práticas corporais (MS, 2006a).

Dessa forma, o Ministério da Saúde, desde a criação da PNPS, por meio de incentivos financeiros possibilitou o desenvolvimento de projetos em 
municípios e estados para implementação de programas de promoção da saúde com ênfase em atividade física (MALTA et al., 2014). Taylor et al. (2004) enfatizam que ações significativas devem ser realizadas não somente pelo governo, mas também por seus gestores políticos para que se criem ambientes nos quais a prática de atividade física seja estimulada ao longo da vida. Como agentes decisivos na gestão do SUS (AGUILERA et al., 2013) e responsáveis pelo bom funcionamento do sistema de saúde oferecido para a população (CECCIM; ARMANI; ROCHA, 2002), os secretários municipais da saúde possuem considerável papel como promotores da atividade física.

A incoerência entre os interesses e as ações realizadas por profissionais e pesquisadores em saúde é uma barreira conhecida para a promoção de atividade física (BROWNSON et al., 2008). Amorin et al. (2013) apontam que a carência de melhor estrutura física e de profissionais qualificados são questões relevantes que devem ser refletidas por gestores municipais. Isso deve ocorrer para que haja um melhor direcionamento de investimentos, a captação de mais parceiros e a efetivação de ações que estejam de acordo com as necessidades da população.

Estudos com o objetivo de conhecer a visão de secretários municipais de saúde e sua concepção sobre a gestão são pontuais e os que existem são de abrangência regional, com número pequeno de participantes (AGUILERA et al., 2013; GRANJA; ZOBOLI; FRACOLLI, 2013; FRACOLLI; GOMES; GRYSCHEK, 2014; LORENZETTI et al., 2014). Envolvendo atividade física, encontrou-se apenas Andrello et al. (2012). Assim, verifica-se a escassez de estudos que relacionem a gestão pública com ênfase em atividade física na visão dos secretários de saúde.

Dessa forma, justifica-se este estudo pelo problema de inatividade física que atinge, principalmente, os idosos; pela relevância dos municípios na busca da minimização desse panorama; da importância do secretário de saúde como gestor da saúde pública e representante social no âmbito municipal e pela necessidade de conhecer o que pode influenciar sua visão sobre a implementação de ações em atividade física.

Assim, tem-se como objetivo do estudo descrever a visão dos secretários municipais de saúde do estado de Santa Catarina sobre as barreiras para desenvolvimento de programas de promoção de atividade física para idosos e associá-las a seu perfil, relacionado aos aspectos sociodemográficos, de formação e de saúde. 
O presente estudo faz parte da pesquisa intitulada "Avaliação de programas de promoção de atividade física no estado de Santa Catarina", desenvolvida pelo Laboratório de Gerontologia (LAGER) do Centro de Ciências da Saúde e do Esporte (CEFID) da Universidade do Estado de Santa Catarina (UDESC) com apoio institucional da Secretaria de Estado de Saúde de Santa Catarina (SES/SC), apoio operacional do Conselho das Secretarias Municipais de Saúde de Santa Catarina (COSEMS/SC), além da parceria institucional da Universidade do Contestado (UnC).

Esse estudo caracteriza-se como exploratório e de corte transversal (GIL, 2010). Foi realizado em Santa Catarina, com os secretários municipais dos 295 municípios do estado. A coleta de dados ocorreu, inicialmente, após a aprovação da SES/SC. Para tanto, o COSEMS/SC disponibilizou aos pesquisadores o banco de dados de contatos de e-mail e telefone das secretarias municipais de saúde e seus gestores que foram contatados para realização do convite a participar da pesquisa. Após o aceite, questionários foram enviados online ou via correspondência, conforme preferência do secretário. Os municípios efetivaram a participação na pesquisa por meio do envio do questionário respondido por seu secretário da saúde.

Todos os secretários municipais de saúde foram convidados a participar do estudo; 6 se recusaram (2 por considerarem as perguntas muito pessoais, 2 por falta de tempo, 1 por problemas pessoais e 1 não apresentou motivo); 85 aceitaram, mas não retornaram os questionários respondidos e 204 aceitaram e retornaram os questionários respondidos, compondo assim a amostra.

Para a coleta de dados foi utilizado um questionário semiestruturado autoaplicável construído com base em instrumentos já utilizados por grupos e laboratórios de pesquisa nacionais e Questionário Internacional de Atividade Física (IPAQ), além de questões específicas elaboradas que foram validadas pela própria pesquisadora. Esse questionário foi dividido em quatro blocos: 1) Aspectos sociodemográficos (GPES, 2015); 2) Aspectos de formação (GPES, 2015); 3) Aspectos relacionados à saúde (LAGER, 2015; MATSUDO et al.; 2001); 4) Barreiras para o desenvolvimento de programas de promoção de atividade física, desenvolvido pela autora.

O bloco número 4, "Barreiras para o desenvolvimento de programas de promoção de atividade física", tem como objetivo verificar a importância que os secretários da saúde atribuem para determinadas barreiras para o desenvolvimento de programas de promoção de atividade física para idosos, para isso, foram elaboradas quatro questões sobre essa temática. As questões 
desse bloco passaram por um processo de validação, um formulário online foi respondido por cinco especialistas (profissionais pesquisadores) da área de atividade física e saúde para que avaliassem a validade, pertinência e clareza; dessa forma, as questões do Bloco 4 foram adaptadas conforme suas sugestões até que chegassem na forma final apresentada no Quadro 1.

Quadro 1 - Variáveis utilizadas nas análises de dados.

\begin{tabular}{|c|c|c|}
\hline Código & $\begin{array}{l}\text { Barreiras } \\
\text { (variáveis dependentes) }\end{array}$ & $\begin{array}{l}\text { Aspectos pessoais } \\
\text { (variáveis independentes) }\end{array}$ \\
\hline B1 & $\begin{array}{l}\text { Profissionais pouco ou nada capacitados para } \\
\text { atender ao público idoso. }\end{array}$ & \multirow{4}{*}{$\begin{array}{l}\text { Aspectos sociodemográficos } \\
\text { Filiação partidária } \\
\text { Sexo } \\
\text { Renda } \\
\text { Estado Civil } \\
\text { Cor } \\
\text { Município possuir programa de AF } \\
\text { Aspectos de formação } \\
\text { Graduação concluída } \\
\text { Graduação na área da saúde } \\
\text { Modalidade da Graduação } \\
\text { Tempo de formação } \\
\text { Pós-Graduação concluída } \\
\text { Pós-Graduação na área da saúde } \\
\text { Aspectos relacionados à saúde } \\
\text { Autorrelato do estado de saúde } \\
\text { Presença de doença crônica } \\
\text { Hábito de fumar } \\
\text { Hábito pregresso de fumar } \\
\text { Nível de atividade física no lazer }\end{array}$} \\
\hline B2 & $\begin{array}{l}\text { Pouco interesse dos idosos da comunidade } \\
\text { em praticar AF. }\end{array}$ & \\
\hline B3 & $\begin{array}{l}\text { Falta de política/diretriz que direcione o } \\
\text { planejamento e implantação de ações/ } \\
\text { atividades relacionadas à AF para idosos. }\end{array}$ & \\
\hline B4 & $\begin{array}{l}\text { Falta de incentivo financeiro para implantação } \\
\text { de ações/atividades relacionadas à AF para } \\
\text { idosos. }\end{array}$ & \\
\hline
\end{tabular}

Fonte: Elaborado pelos autores, 2016.

Para as barreiras, foram adotadas as opções de respostas: "extremamente importante", "muito importante", "importante", "pouco importante" e "nenhuma importância". Para fins estatísticos, as categorias foram agrupadas em "maior importância" (extremamente importante e muito importante) e "menor importância" (importante, pouco importante e nenhuma importância).

O presente estudo foi enviado e aprovado pelo Comitê de Ética Envolvendo Seres Humanos (CEP) da UDESC sob protocolo CAAE: 7414515.0.0000.0118. Para a realização dessa pesquisa, foram cumpridos os princípios éticos de acordo com a resolução 466/12 do Conselho Nacional de Saúde (CNS). O processo de coleta de dados ocorreu conforme apresentado na Figura 1. 
Figura 1 - Processo e período de coleta de dados.

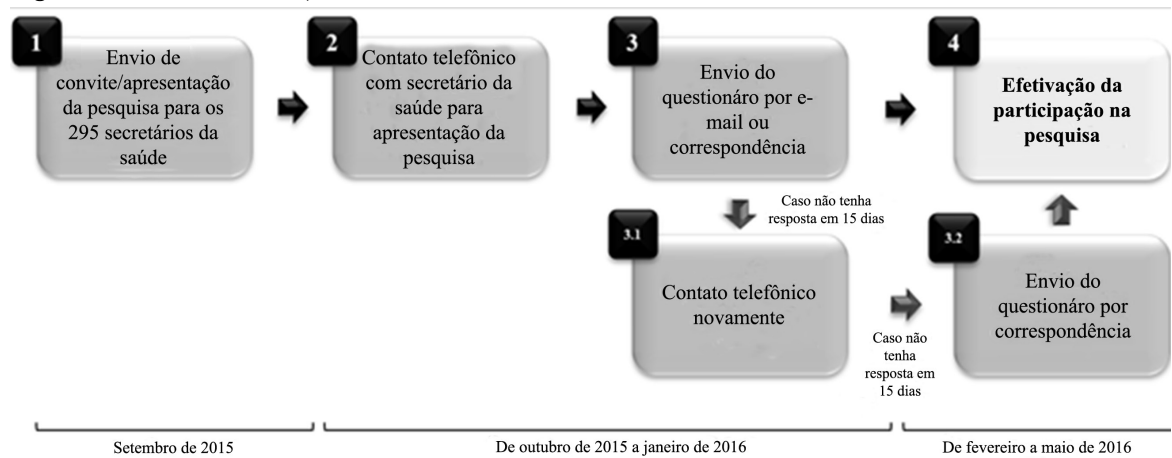

Fonte: Elaborada pelos autores, 2016.

Os dados foram digitalizados e analisados com o auxílio do software estatístico IBM SPSS ${ }^{\circledR}$, versão 20.0. Inicialmente, todas as variáveis foram analisadas descritivamente por meio de frequência simples e relativa. Na análise inferencial foram utilizados os testes Qui-quadrado ou Exato de Fisher para associação univariada entre as características dos secretários municipais de saúde e as questões relacionadas às barreiras para desenvolvimento de programas de promoção de atividade física para idosos. A associação multivariada foi analisada por meio de regressão logística binária, com a entrada das variáveis realizada pelo método stepwise forward, estimando-se razão de chances (RC) e intervalos de confiança (IC95\%). Além da análise de regressão bruta, foram desenvolvidos quatro modelos de análise ajustada para verificar a magnitude de associação entre as respostas relacionadas às barreiras e as características dos secretários de saúde. Realizaram-se ajustes para as variáveis que apresentaram $p \leq 0,25$. Adotou-se um nível de significância de 5\%.

\section{Resultados}

Ressalta-se que os resultados apresentados possuem números diferentes de respostas devido ao fato de alguns secretários municipais de saúde de Santa Catarina não terem respondido todas as perguntas do questionário.

Participaram do estudo secretários municipais de saúde do estado de Santa Catarina, sendo 103 (51\%) homens e 99 (49\%) mulheres. A média de idade da amostra foi de 43,7 9,9 anos (mínimo 25 e máximo 68 anos). Os gestores em saúde, em sua maioria, atribuíram maior importância para as barreiras "Profissionais pouco ou nada capacitados para atender ao público idoso" e "Falta 
de incentivo financeiro para implantação de ações/atividades relacionadas à AF para idosos"; no entanto, a prevalência de maior importância também foi alta nas demais barreiras, conforme observa-se na Tabela 1.

Tabela 1 - Análise de frequência de barreiras percebidas pelos secretários municipais de saúde de Santa Catarina para o desenvolvimento de programas de atividade física para idosos $(n=203)$.

\begin{tabular}{llll}
\hline Cód. & Barreiras & $\begin{array}{l}\text { Menor } \\
\text { importância } \\
\mathbf{f ( \% )}\end{array}$ & $\begin{array}{l}\text { Maior } \\
\text { importância } \\
\mathbf{f}(\%)\end{array}$ \\
\hline B1 & $\begin{array}{l}\text { Profissionais pouco ou nada capacitados para } \\
\text { atender ao público idoso }\end{array}$ & $96(47,3)$ & $107(52,7)$ \\
B2 & $\begin{array}{l}\text { Pouco interesse dos idosos da comunidade em } \\
\text { praticar AF }\end{array}$ & $104(51,2)$ & $99(48,8)$ \\
B3 & $\begin{array}{l}\text { Falta de política/diretriz que direcione o } \\
\text { planejamento e implantação de ações/atividades } \\
\text { relacionadas à AF para idosos }\end{array}$ & $105(51,7)$ & $98(48,3)$ \\
B4 & $\begin{array}{l}\text { Falta de incentivo financeiro para implantação de } \\
\text { ações/atividades relacionadas à AF para idosos }\end{array}$ & $78(38,4)$ & $125(61,6)$ \\
\hline
\end{tabular}

Legenda: Cód = código; $\mathrm{AF}$ = atividade física; $\mathrm{f}$ = frequência simples; \% = frequência relativa.

Fonte: Elaborada pelos autores, 2016.

Com relação à magnitude da associação entre as barreiras e os aspectos sociodemográficos, de formação e de saúde, a regressão logística binária mostrou (Tabela 2), na análise ajustada, que os gestores municipais que vivem sem companheiro(a) apresentam 2,5 vezes mais chances de considerar a barreira "Profissionais pouco ou nada capacitados para atender ao público idoso" muito importante do que aqueles que vivem acompanhados. Também verificou-se que indivíduos ativos fisicamente no lazer têm 4,8 vezes mais chances de considerarem essa barreira muito importante quando comparados com os inativos no mesmo domínio.

Com relação à barreira "Pouco interesse dos idosos da comunidade em praticar atividade física", constatou-se que o fato do município possuir programa de atividade física, não necessariamente exclusivo para idosos, aumenta em 2,6 vezes as chances de seus secretários municipais de saúde considerarem muito importante essa barreira. Também foi verificado que secretários que vivem sem companheiro(a) possuem 2 vezes mais chances de atribuírem maior importância à mesma barreira.

Além disso, constatou-se que gestores municipais em saúde que vivem sem companheiro(a) possuem 2,9 vezes mais chances de considerarem a barreira "Falta de política/diretriz que direcione o planejamento e implantação de ações/ atividades relacionadas à atividade física para idosos" como muito importante. 
Já os secretários que autorrelataram possuir pelo menos uma doença crônica apresentam $70 \%$ mais chances de não considerarem a barreira "Falta de incentivo financeiro para implantação de ações/atividades relacionadas à atividade física para idosos" como possuindo maior importância. Ainda relacionado a essa barreira, indivíduos que vivem sem companheiro(a) têm 2,3 vezes mais chances e os que possuem renda de mais de 10 salários mínimos, $80 \%$ mais chances de não considerar muito importante.

Tabela 2 - Análise multivariada entre barreiras percebidas pelos secretários municipais de saúde de Santa Catarina para o desenvolvimento de programas de atividade física para idosos e as características dos mesmos.

\begin{tabular}{|c|c|c|c|c|c|c|}
\hline \multicolumn{7}{|c|}{ B1 } \\
\hline \multirow[t]{2}{*}{ Exposições } & \multicolumn{3}{|c|}{ Análise bruta } & \multicolumn{3}{|c|}{ Análise ajustada } \\
\hline & $\mathrm{RC}$ & $p$ & IC95\% & $\mathrm{RC}$ & $p$ & IC95\% \\
\hline \multicolumn{7}{|l|}{ Estado Civil } \\
\hline Com companheiro(a) & 1 & 0,007 & $1,3-5,1$ & 1 & $0,009^{*}$ & $1,2-5,2$ \\
\hline Sem companheiro(a) & 2,5 & & & 2,5 & & \\
\hline \multicolumn{7}{|l|}{ Nível de AF } \\
\hline Inativo & 1 & & & 1 & & \\
\hline Insuficientemente ativo & 4,3 & 0,006 & $1,5-12,2$ & 1,5 & 0,183 & $0,8-3,1$ \\
\hline Ativo & 4,8 & 0,009 & $1,4-15,9$ & 2,7 & $0,025^{\star}$ & $1,1-6,4$ \\
\hline \multicolumn{7}{|l|}{ Sexo } \\
\hline Masculino & 1 & 0,059 & $0,3-1,0$ & 1 & 0,153 & $0,3-1,1$ \\
\hline Feminino & 0,5 & & & 0,6 & & \\
\hline \multicolumn{7}{|c|}{$\mathrm{B} 2$} \\
\hline \multirow[t]{2}{*}{ Exposições } & \multicolumn{3}{|c|}{ Análise bruta } & \multicolumn{3}{|c|}{ Análise ajustada } \\
\hline & $\mathrm{RC}$ & $p$ & IC95\% & $\mathrm{RC}$ & $p$ & IC95\% \\
\hline \multicolumn{7}{|l|}{$\begin{array}{l}\text { Município possui } \\
\text { programa }\end{array}$} \\
\hline Não & 1 & 0,002 & $1,4-4,8$ & 1 & $0,004^{*}$ & $1,3-5,2$ \\
\hline $\operatorname{Sim}$ & 2,6 & & & 2,6 & & \\
\hline \multicolumn{7}{|l|}{ Estado Civil } \\
\hline Com companheiro(a) & 1 & 0,038 & $1,0-3,8$ & 1 & $0,030^{\star}$ & $1,0-4,5$ \\
\hline Sem companheiro(a) & 2,0 & & & 2,2 & & \\
\hline \multicolumn{7}{|l|}{$\begin{array}{l}\text { Hábito pregresso } \\
\text { de fumar }\end{array}$} \\
\hline Sim & 1 & 0,255 & $0,3-1,3$ & 1 & 0 120 & 021 \\
\hline Não & 0,6 & & & 0,5 & 0,120 & $0,2-1,1$ \\
\hline
\end{tabular}




\section{B3}

\begin{tabular}{|c|c|c|c|c|c|c|}
\hline \multirow[t]{2}{*}{ Exposições } & \multicolumn{3}{|c|}{ Análise bruta } & \multicolumn{3}{|c|}{ Análise ajustada } \\
\hline & $\mathrm{RC}$ & $\mathrm{p}$ & IC95\% & $\mathrm{RC}$ & $\mathrm{p}$ & IC95\% \\
\hline \multicolumn{7}{|l|}{ Estado Civil } \\
\hline Com companheiro(a) & 1 & 0,002 & $1,4-5,7$ & 1 & $0,001^{*}$ & $1,7-7,2$ \\
\hline Sem companheiro(a) & 2,9 & & & 3,5 & & \\
\hline \multicolumn{7}{|l|}{ Presença de doença } \\
\hline Não & 1 & 0,106 & $0,2-1,1$ & 1 & 0,069 & $0,1-1,0$ \\
\hline Sim & 0,4 & & & 0,4 & & \\
\hline
\end{tabular}

\section{Graduação concluída}

$\begin{array}{llllll}\text { Não } & 1 & 0,125 & 0,3-1,1 & 1 & 0,113 \\ \text { Sim } & 0,6 & & & 0,3-1,1\end{array}$

\section{Renda}

\begin{tabular}{|c|c|c|c|c|c|c|}
\hline Até 4 SM & 1 & & & 1 & & \\
\hline De 4 a 10 SM & 0,7 & 0,314 & $0,3-1,3$ & 0,6 & 0,160 & $0,3-1,1$ \\
\hline Mais de $10 \mathrm{SM}$ & 0,3 & 0,086 & $0,1-1,1$ & 0,3 & 0,078 & $0,1-1,1$ \\
\hline \multicolumn{7}{|c|}{ B4 } \\
\hline \multirow[t]{2}{*}{ Exposições } & \multicolumn{3}{|c|}{ Análise bruta } & \multicolumn{3}{|c|}{ Análise ajustada } \\
\hline & $\mathrm{RC}$ & $p$ & IC95\% & $\mathrm{RC}$ & $p$ & IC95\% \\
\hline \multicolumn{7}{|l|}{ Presença de doença } \\
\hline Não & 1 & 0,007 & $0,1-0,7$ & 1 & $0,007^{*}$ & $0,1-0,7$ \\
\hline Sim & 0,3 & & & 0,2 & & \\
\hline \multicolumn{7}{|l|}{ Estado Civil } \\
\hline Com companheiro(a) & 1 & 0,020 & $1,1-4,8$ & 1 & $0,008^{*}$ & $1,3-6,3$ \\
\hline Sem companheiro(a) & 2,3 & & & 2,9 & & \\
\hline \multicolumn{7}{|l|}{ Renda } \\
\hline Até 4 SM & 1 & & & 1 & & \\
\hline De 4 a 10 SM & 0,7 & 0,321 & $0,3-1,3$ & 0,7 & 0,396 & $0,3-1,4$ \\
\hline Mais de $10 \mathrm{SM}$ & 0,2 & 0,029 & $0,1-0,8$ & 0,2 & $0,016^{*}$ & $0,1-0,7$ \\
\hline \multicolumn{7}{|l|}{ Sexo } \\
\hline Masculino & 1 & 0,185 & $0,3-1,2$ & 1 & 0,278 & $0,3-1,3$ \\
\hline Feminino & 0,6 & & & 0,7 & & \\
\hline
\end{tabular}

Legenda: $\mathrm{AF}$ = atividade física; $\mathrm{SM}$ = salários mínimos; $\mathrm{RC}$ = razão de chance; $\mathrm{IC}$ = intervalo de confiança.

Fonte: Elaborada pelos autores, 2016. 
As discussões dos resultados do presente estudo serão apresentadas de acordo com as barreiras para o desenvolvimento de programas de promoção de atividade física, conforme a visão dos secretários municipais de saúde do estado de Santa Catarina.

\subsection{Profissionais pouco ou nada capacitados para atender ao público idoso}

Em análise descritiva, foi verificado que a maior parte dos secretários municipais de saúde do estado de Santa Catarina considerou a barreira "Profissionais pouco ou nada capacitados para atender ao público idoso" com maior grau de importância. Apesar da adequação de currículos para que se incluam temas relacionados ao envelhecimento humano nos cursos de formação de profissionais da saúde ser preconizado pela Política Nacional de Saúde da Pessoa Idosa (MS, 2006b); Carvalho e Hennington (2015), em revisão integrativa, verificaram que o envelhecimento como disciplina obrigatória nos cursos de graduação em educação física ainda é escasso. Benedetti, Gonçalves e Mota (2007) enfatizam que os recursos humanos profissionais alocados para atuação em ações de atividade física para idosos devem ser devidamente capacitados e especializados, a fim de cumprir com as metas da promoção do envelhecimento saudável.

Em pesquisa realizada com 415 secretários municipais de saúde de todas as unidades da federação, foi verificado que esses gestores consideram que a desqualificação dos profissionais de saúde é um problema que acreditam influenciar diretamente na qualidade da gestão dos sistemas locais ou municipais de saúde (MS, 2006c). Outro problema apontado no mesmo estudo é a dificuldade de contratação de pessoal e precarização dos vínculos empregatícios, além da grande rotatividade dos trabalhadores da saúde. Jorge Castro, Janete Castro e Vilar (2005), Franco (2011) e Garózi (2014) também relataram que os secretários de saúde entrevistados em seus estudos apontaram a falta de capacitação profissional como um fator que dificulta a gestão municipal.

Dessa forma, percebe-se que a administração dos recursos humanos em saúde é um problema bastante salientado independentemente na área e/ou nível de atenção à saúde. Essa dificuldade pode ter, inclusive, relação direta com as contínuas mudanças entre dirigentes das Secretarias Municipais de Saúde (SMS), o que, acarreta desgaste e descontinuidade nas ações, nos serviços e nos programas de saúde, dificultando a gestão dos sistemas locais ou municipais de saúde (MS, 2006c). 
Por outro lado, profissionais de educação física de Santa Catarina apontaram a baixa remuneração como principal barreira para atuação na área (SALLES; FARIAS; NASCIMENTO, 2015). Uma remuneração adequada é importante para o trabalhador, pois demonstra o reconhecimento do trabalho realizado (PRESTES et al., 2016). Assim, o baixo salário pode ser um fator desmotivador para maior interesse em capacitações por parte desses profissionais. Sugere-se que estratégias sejam criadas a fim de aproximar o trabalhador das esferas de gestão pública em saúde. Dessa forma, medidas poderão ser tomadas para suprir as barreiras apontadas por ambos os lados com o intuito de potencializar o trabalho com relação à gestão e prática de promoção de atividade física nos municípios.

Ainda com relação à barreira "Profissionais pouco ou nada capacitados para atender ao público idoso", foi verificado que secretários da saúde ativos possuem mais chances de considerá-la mais importante. Estudo de base populacional ocorrido em Florianópolis, capital catarinense, constatou que o tipo de atividade física realizada no lazer com maior prevalência é a caminhada (32,9\%); no entanto, as demais modalidades citadas (como musculação, ginástica, natação, entre outros) normalmente são realizadas com supervisão profissional (DEL DUCA et al., 2014). Mendes et al. (2010) destacam a relevância do profissional de educação física como fonte de informação sobre a importância da atividade física. Dessa forma, pode-se considerar que os secretários municipais de saúde de Santa Catarina, ativos fisicamente no lazer, possuem maior acesso a profissionais da área da atividade física os quais podem transmitir informações sobre a importância e a forma que a atividade física deve ser prescrita; os fazendo, assim, considerarem essa barreira com maior importância.

\subsection{Pouco interesse dos idosos da comunidade em praticar atividade física}

Apesar de não representar a maioria, muitos secretários consideraram maior importância nessa barreira. Conforme o envelhecimento há uma tendência do aumento da inatividade física (HALLAL et al., 2012) Estudo de base populacional realizado em Florianópolis verificou que 31,5\% dos idosos são ativos fisicamente no lazer (SANTOS et al., 2015). Possuir 80 anos ou mais, possuir dor crônica e histórico de queda no último ano diminuem as chances de idosos realizaram atividade física (LIMA et al., 2015). Além disso, os principais motivos considerados por idosos para ingresso em programas de promoção de atividade física são relacionados à saúde (RIBEIRO et al., 2012; MAZO et al., 2013; SANDRESCHI; PETREÇA; MAZO, 2014). 
Ribeiro et al. (2012) explicam que além da necessidade de vontade própria, o idoso ainda depende de toda a interação que faz com o ambiente para que se sinta motivado a participar de programas de atividade física. Uma infraestrutura adequada e suporte social aumentam a prevalência da prática de atividade física no lazer (GIEHL et al., 2012). Assim, cabe aos gestores públicos a reflexão sobre a motivação da considerada falta de interesse dos idosos em praticar atividades físicas e, por conseguinte, a busca por incentivos e adequações ambientais para que o interesse desse público aumente visando a prevenção de doenças e a melhoria da sua qualidade de vida.

Sobre a mesma barreira "Pouco interesse dos idosos da comunidade em praticar atividade física", foi constatado que o fato de o município possuir programa de atividade física, não necessariamente exclusivo para idosos, aumenta as chances de seus secretários municipais de saúde considerá-la muito importante. Os gestores de municípios que promovem atividade física para a população por meio de programas podem ter uma visão mais direta do interesse de idosos em participarem desses programas.

\subsection{Falta de política/diretriz que direcione o planejamento e implantação de ações/atividades relacionadas à atividade física para idosos"}

Essa barreira foi relatada como possuindo maior importância por quase metade dos secretários de saúde. No entanto, muitas estratégias de promoção da atividade física vêm sendo incluídas em planos referentes à saúde da população (HEATH et al., 2012). Inicialmente a OMS propôs a Estratégia Global de Alimentação Saudável, Atividade Física e Saúde (OMS, 2004); no Brasil, desde 2005 o Ministério da Saúde busca, por meio de políticas públicas e criação de projetos, incentivar um comportamento mais ativo fisicamente e com alimentação mais saudável da população (MALTA et al., 2009). Essas estratégias tornaram-se mais incisivas em 2006, com a publicação da Política Nacional de Promoção da Saúde (PNPS) com a inclusão da atividade física/ práticas corporais dentre as áreas temáticas existentes (MS, 2006a). Dessa forma, o Ministério da Saúde impulsionou a promoção de atividade física como forma prevenção de doenças atuando na atenção primária à saúde.

Com relação específica a idosos, a Política Nacional de Saúde da Pessoa Idosa (PNSPI) preconiza que se deve "facilitar a participação das pessoas idosas em equipamentos sociais, grupos de terceira idade, atividade física, conselhos de saúde locais e conselhos comunitários onde o idoso possa ser 
ouvido e apresentar suas demandas e prioridades" (MS, 2006b). Além disso, os idosos aparecem como grupo prioritário no Plano de Ações Estratégicas para o Enfrentamento das Doenças Crônicas Não Transmissíveis (DCNT) (MS, 2011). Benedetti, Gonçalves e Mota (2007) realizaram uma proposta de política pública de atividade física para idosos e argumentaram que o Brasil possui potencial para implementação de bons programas, faltando apenas incentivo político para que isso se concretize. Marin e Panes (2015) reconhecem essa evolução nas políticas de promoção à saúde do idoso e ressaltam a importância da legislação. Uma vez que ela expõe a necessidade de enfrentamento da situação e gera abertura para reflexões e mudanças que podem ser realizadas por governantes.

Dessa forma, apensar de mais escassas, políticas/diretrizes para planejamento e implementação de ações/atividades relacionadas a idosos existem. Além disso, aquelas formuladas para a população geral podem ser utilizadas e adaptadas para que atendam as particularidades desse público. Assim, percebe-se a necessidade de que essas informações cheguem aos secretários municipais de saúde. Essa necessidade já foi apontada pelos próprios secretários de saúde que consideraram a melhoria da comunicação entre o Ministério da Saúde, os estados e os municípios como um fator que contribuiria para melhorar a gestão em seu município (MS, 2006c).

\subsection{Falta de incentivo financeiro para implantação de ações/atividades relacionadas à atividade físicas para idosos}

Essa barreira foi a que apresentou mais prevalência de maior importância atribuída pelos secretários da saúde. A escassez de recursos financeiros como um problema de gestão pública municipal é amplamente citada por secretários da saúde de diversas regiões do país (CASTRO, Jorge; CASTRO, Janete; VILAR, 2005; MS, 2006c; FRANCO, 2011; GAROZI, 2014).

Apesar de considerarem a falta de recursos financeiros como a barreira com mais prevalência de maior importância, em Santa Catarina 40 municípios e a Secretaria de Estado da Saúde (SES/SC) foram contemplados com editais/ portarias da Rede Nacional de Atividade Física em diferentes períodos totalizando R \$ 4.339.153,72 disponibilizados. Quanto ao programa Academia da Saúde, presente em 122 municípios, o investimento do Ministério da Saúde do Brasil previsto para Santa Catarina é de R \$16.360.000,00 em 2016, o valor é variável devido ao fato do repasse da verba ainda estar em andamento (PETREÇA et al., 2017). 
Entende-se que o financiamento citado não é destinado para ações que atendam exclusivamente idosos; no entanto, as ações desenvolvidas podem e devem incluir atividades que atendam esse público de acordo com suas necessidades específicas. Verifica-se que existem oportunidades para promoção da saúde com ênfase em atividade física de financiamento no Brasil e cabe aos gestores localizá-las, gerenciá-las e implementá-las com base em políticas federais existentes.

Verificou-se no presente estudo que os secretários que possuíam pelo menos uma doença crônica apresentam 70\% mais chances de não considerarem essa barreira como possuindo maior importância. $\mathrm{O}$ fato de ter doença aproxima o indivíduo dos serviços de saúde (SILVA et al., 2011) e pode melhorar sua percepção das necessidades e importância de investimentos financeiros em outras áreas da saúde, além da atenção primária. Dessa forma, podem considerar que os recursos já existentes para AF sejam suficientes tendo em vista a necessidade de repasse para outros níveis de atenção à saúde.

No presente estudo foi verificado que o fato do secretário municipal de saúde possuir uma renda mensal de mais de 10 salários apresenta $80 \%$ as chances de não considerar muito importante essa barreira. Isso pode ocorrer pelo fato de secretários de municípios de grande porte receberem remuneração mais alta do que aqueles de municípios menores, a mesma relação ocorre com o repasse de recursos vindos do governo estadual e federal (BRASIL, 2004; MS, 2016b). Dessa forma, indicando que secretários que possuem salários mais altos, possuem mais recursos financeiros em sua secretaria e, consequentemente, possuem menos chances de percebem essa barreira como maior importância.

Além dos resultados já apresentados, no presente estudo foi verificado que viver sem companheiro se associou ao aumento de chance de os secretários municipais de saúde considerarem mais importante as quatro barreiras estudadas no presente estudo. Existe uma lacuna no conhecimento científico acerca de pesquisas que possam justificar e estarem relacionados com esse fato. Sugere-se que futuros estudos, principalmente de cunho qualitativo, abordem essa temática de forma mais aprofundada para verificar a relação da situação conjugal com percepções sobre o ambiente de trabalho.

\section{Conclusão}

No presente estudo foi possível verificar que as barreiras consideradas mais importantes pelos secretários municipais de saúde de Santa Catarina (SC) foram “Falta de incentivo financeiro para implantação de ações/atividades 
relacionadas à atividade físicas para idosos" e "Profissionais pouco ou nada capacitados para atender ao público idoso", consecutivamente.

$\mathrm{O}$ fato dos gestores(as) viverem sem companheiro(a) aumenta as chances de considerarem todas as barreiras mais importantes. Ser ativo fisicamente no lazer também aumenta as chances de considerar "Profissionais pouco ou nada capacitados para atender ao público idoso" mais importante; aqueles que atuam em municípios que possuem programas de atividade física, não necessariamente exclusivo para idosos possuem mais chances de considerar a barreira "Pouco interesse dos idosos da comunidade em praticar atividade física" como muito importante; quanto à barreira "Falta de incentivo financeiro para implantação de ações/atividades relacionadas à atividade física para idosos" contatou-se que os secretários que autorrelataram possuir pelo menos uma doença crônica e aqueles que possuem renda elevada apresentam mais chances de não considerarem essa barreira com maior importância.

Por meio dos resultados do presente estudo espera-se que reflexões sejam realizadas a fim de minimizar as barreiras consideradas pelos secretários da saúde. Além disso, sugere-se que futuros estudos sejam realizados com o intuito de verificar as sugestões desses gestores para que a promoção de atividade física para idosos seja mais efetiva em seus municípios.

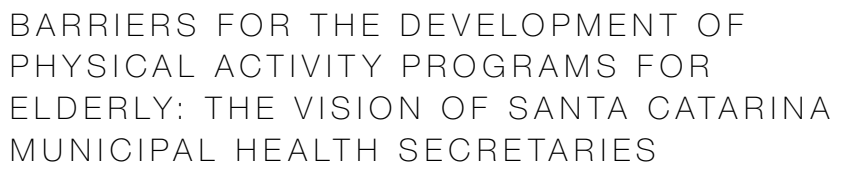

Objective: to describe the Santa Catarina (SC) municipal health secretaries (MHS) perception on barriers to the development of physical activity (PA) promotion programs for elderly people and to associate them with the characteristics of their profile. Method: participated in the study 204 (MHS). A questionnaire was applied with sociodemographic, training, and health issues and four barriers to the development of PA programs for the elderly, which should assign a degree of importance. Results: the main barrier considered by MHS was "lack of financial incentive to implement actions/activities related to PA for the elderly" and was associated with marital status, the level of physical activity of the secretaries and the presence of program of AF in the city. Conclusion: the MHS considers that the lack of financial 
keywords

Public Health. Health Services Administration. Health Manager.

\author{
referências
}

AGUILERA, Sandra Lúcia et al. Articulação entre os níveis de atenção dos serviços de saúde na Região Metropolitana de Curitiba: desafios para os gestores. Revista de Administração Pública, Rio de Janeiro, v. 47, n. 4, p. 1021-1040, 2013.

AMORIM, Tales et al. Descrição dos programas municipais de promoção da atividade física financiados pelo Ministério da Saúde. Revista Brasileira de Atividade Física \& Saúde, Pelotas, v. 18, n. 1, p. 63-74, 2013

ANDRELLO, Eduardo et al. Atividade física e saúde pública sob o olhar de secretários municipais de saúde. Revista Brasileira de Atividade Física \& Saúde, Pelotas, v. 17, n. 3, p. 206-211, 2012.

BENEDETTI, Tânia; GONÇALVES, Lúcia; MOTA, Jorge. Uma proposta de política pública de atividade física para idosos. Texto e Contexto - Enfermagem, Florianópolis, v. 16, n. 3, p. 387-398, 2007

BRASIL. Ministério da Saúde. Sistema Nacional de Auditoria do SUS - SNA. Brasília, DF: MS, 2004

BROWNSON, Ross et al. Environmental and policy approaches for promoting physical activity in the United States: a research agenda. Journal of Physical Activity and Health, Birmingham, v. 5, n. 4, p. 488-503, 2008.

CARVALHO, Claudia; HENNINGTON, Élida. A abordagem do envelhecimento na formação universitária dos profissionais de saúde: uma revisão integrativa. Revista Brasileira de Geriatria e Gerontologia, Rio de Janeiro, v. 18, n. 2, p. 417-431, 2015.

CASTRO, Janete; CASTRO, Jorge; VILAR, Rosana. A. Quem são os Gestores Municipais de Saúde no Rio Grande do Norte? Um estudo sobre o perfil. Natal: NESC/UFRN, 2005.

CECCIM, Ricardo; ARMANI, Teresa; ROCHA, Cristianne. O que dizem a legislação e o controle social em saúde sobre a formação de recursos humanos e o papel dos gestores públicos, no Brasil. Ciência \& Saúde Coletiva, Rio de Janeiro, v. 7, n. 2, p. 373-383, 2002.

DEL DUCA, Giovâni et al. Atividades físicas no lazer entre adultos de Florianópolis, Santa Catarina, Brasil: estudo populacional sobre as características das práticas e de seus praticantes. Ciência \& Saúde Coletiva, Rio de Janeiro, v. 19, n. 11, p. 4595-4604, 2014.

FRACOLLI, Lislaine; GOMES, Maria; GRYSCHEK, Anna. Percepções de gestores municipais sobre ações de promoção da saúde: em foco os agentes comunitários de saúde. Saúde e Sociedade, São Paulo, v. 23, n. 3, p. 919-927, 2014.

FRANCO, Adriana. Gestão municipal de saúde: limites e possibilidades de gestão na percepção dos secretários de saúde da AMREC. 2011. Monografia (Especialização em Gestão Empresarial) - Universidade do Extremo Sul Catarinense, Criciúma, 2011.

GARÓZI, Denise. Perfil do gestor municipal da saúde e a percepção da Estratégia Saúde da Família - DRS IX - Marília/SP. 2014. 95 f. Dissertação (Mestrado em Saúde 
Coletiva) - Faculdade de Medicina, Universidade Estadual Paulista Júlio de Mesquita Filho, Botucatu, 2014.

GIEHL, Maruí et al. Atividade física e percepção do ambiente em idosos: estudo populacional em Florianópolis. Revista de Saúde Pública, São Paulo, v. 46, n. 3, 2012.

GIL, Antonio. Como elaborar projetos de pesquisa. 5. ed. São Paulo: Atlas, 2010.

GRANJA, Gabriela; ZOBOLI, Elma; FRACOLLI Lislaine. A. O discurso dos gestores sobre a equidade: um desafio para o SUS. Ciência \& Saúde Coletiva, Rio de Janeiro, v. 18, n. 12, p. 3759-3764, 2013

GRUPO DE PESQUISA EM ESTILO DE VIDA E SAÚDE (GPES). Projeto SUS + Ativo. Recife: GPES, 2015. Disponível em: http://www.gpesupe.org/projetos.php. Acesso em: 26 mar. 2015.

HALLAL, Pedro et al. Global physical activity levels: surveillance progress, pitfalls, and prospects for the Lancet Physical Activity Series Working Group. The Lancet, London, v. 380, n. 9838, p. 247-257, jul. 2012.

HEATH, Gregory et al. Evidence-based intervention in physical activity: lessons from around the world. The Lancet, London, v. 380, n. 9838, p. 272-281, 2012.

LABORATÓRIO DE GERONTOLOGIA (LAGER). Instrumentos mais utilizados. Disponível em: https://www.udesc.br/cefid/lager. Acesso em: 23 jul. 2019.

LIMA, Alisson et al. Fatores associados à atividade física em idosos de Estação, Rio Grande do Sul: estudo de base populacional. Revista Brasileira de Atividade Física e Saúde, Florianópolis, v. 20, n. 6, 2015.

LORENZETTI, Jorge et al. Gestão em saúde no brasil: diálogo com gestores públicos e privados. Texto e Contexto - Enfermagem, Florianópolis, v. 23, n. 2, p. 417-425, 2014.

MALTA, Deborah et al. A implementação das prioridades da Política Nacional de Promoção da Saúde, um balanço, 2006 a 2014. Ciência e Saúde Coletiva, Rio de Janeiro, v. 19, n. 11, p. 4301-4312, 2014.

MALTA, Deborah et al. A Politica Nacional de promoção da saúde e a agenda da atividade física no contexto do SUS. Epidemiologia e Serviços de Saúde, Brasília, DF, v. 18, n. 1, p. 79-86, 2009.

MARIN, Maria; PANES, Vanessa. Envelhecimento da população e as políticas públicas de saúde. Revista do Instituto de Políticas Públicas de Marília, Marília, v. 1, n. 1, 2015.

MATSUDO, Sandra et al. Questionário Internacional de Atividade Física (IPAQ): estudo da validade e reprodutibilidade no Brasil. Revista Brasileira de Atividade Física e Saúde, Florianópolis, v. 6, n. 2, 2001.

MAZO, Giovana et al. Grupo de Estudos da Terceira Idade - GETl: uma proposta de integração entre extensão, ensino e pesquisa voltados à pessoa idosa. Revista Conexão UEPG, Ponta Grossa, v. 9, n. 1, p. 94-105, 2013.

MENDES, Márcio et al. Fontes de informações sobre a importância da atividade física: estudo de base populacional. Revista Brasileira de Atividade Física e Saúde, Florianópolis, v. 15, n. 3, 2010.

MINISTÉRIO DA SAÚDE (MS). Agência Nacional de Saúde Suplementar. Vigitel Brasil 2014 - Saúde Suplementar: vigilância de fatores de risco e proteção para doenças crônicas por inquérito telefônico. Brasília, DF: MS, 2015.

MINISTÉRIO DA SAÚDE (MS). Portaria n 687, de 30 de março de 2006. Aprova a Política de Promoção da Saúde. Brasília, DF: MS, 2006a.

MINISTÉRIO DA SAÚDE (MS). Portaria n².528, de 19 de outubro de 2006. Aprova a Política Nacional de Saúde da Pessoa Idosa. Brasília, DF: MS 2006b. 
MINISTÉRIO DA SAÚDE (MS). Secretaria-Executiva. Departamento de Apoio à Descentralização. Gestores do SUS: olhares e vivências. Brasília, DF: Editora MS, 2006c.

MINISTÉRIO DA SAÚDE (MS). Secretaria de Vigilância em Saúde. Plano de ações estratégicas para o enfrentamento das doenças crônicas não transmissíveis (DCNT) no Brasil 2011-2022. Brasília, DF: MS, 2011.

ORGANIZAÇ̃̃O MUNDIAL DA SAÚDE (OMS). Estratégia Global em Alimentação Saudável, Atividade Física e Saúde. In: ASSEMBLEIA MUNDIAL DE SAÚDE, 57., 2004, Brazzaville. Anais [...]. Brazzaville: OMS, 2004.

PRESTES, Francine et al. Danos à saúde dos trabalhadores de enfermagem em um serviço de hemodiálise. Revista Gaúcha de Enfermagem, Porto Alegre, v. 37, n. 1, 2016.

PETREÇA, Daniel et al. Avaliação de intervenções em atividade física para idosos e outras populações na saúde pública de Santa Catarina. 2017. Tese (Doutorado em Ciências do Movimento Humano) - Centro de Ciências da Saúde e do Esporte, Universidade do Estado de Santa Catarina, Florianópolis, 2017.

RIBEIRO, José et al. Adesão de idosos a programas de atividade física: motivação e significância. Revista Brasileira de Ciências do Esporte, Porto Alegre, v. 34, p. 4, 2012.

SALLES, William; FARIAS, Gelcemar; NASCIMENTO, Juarez. Inserção profissional e formação continuada de egressos de cursos de graduação em Educação Física. Revista Brasileira de Educação Física e Esporte, São Paulo, v. 29, n. 3, p. 475-486, 2015.

SANDRESCHI, Paula; PETREÇA, Daniel; MAZO, Giovana. Avaliação de um programa universitário de atividade física para idosos pelo modelo RE-AlM. Revista Brasileira de Atividade Física e Saúde, Florianópolis, v. 20, n. 3, 2015

SANTOS, Franco et al. Prevalência de dor crônica e sua associação com a situação sociodemográfica e atividade física no lazer em idosos de Florianópolis, Santa Catarina: estudo de base populacional. Revista Brasileira de Epidemiologia, São Paulo, v. 18, n. 1, p. 234-247, 2015

SILVA, Zilda et al. Perfil sociodemográfico e padrão de utilização dos serviços de saúde do Sistema Único de Saúde (SUS). Ciência \& Saúde Coletiva, Rio de Janeiro, v. 16, n. 9, p. 3807-3816, 2011.

SUN, Fei; NORMAN, lan; WHILE, Alison. Physical activity in older people: a systematic review. BMC Public Health, New York, v. 13, n. 1, p. 449, 2013.

TAYLOR, Adrian et al. Physical activity and older adults: a review of health benefits and the effectiveness of interventions. Journal of Sports Sciences, London, v. 22, n. 8, p. 703-725, 2004. 